\title{
Direct-Write Drawing of Carbon Nanotube/Polymer Composite Microfibers
}

\author{
Scott M. Berry, ${ }^{1,2,3}$ Santosh Pabba, ${ }^{2}$ Robert W. Cohn, ${ }^{2}$ and Robert S. Keynton ${ }^{1,2,4}$ \\ ${ }^{1}$ Department of Mechanical Engineering, University of Louisville, 200 Sacket Hall, Louisville, KY 40292, USA \\ ${ }^{2}$ The Electrooptics Research Institute and Nanotechnology Center, University of Louisville, Louisville, KY 40292, USA \\ ${ }^{3}$ Department of Biomedical Engineering, University of Wisconsin-Madison, 6036 WIMR, 1111 Highland Avenue, Madison, \\ WI 53705, USA \\ ${ }^{4}$ Department of Bioengineering, University of Louisville, 419 Lutz Hall, Louisville, KY 40292, USA
}

Correspondence should be addressed to Scott M. Berry, scott.michael.berry@gmail.com

Received 30 April 2012; Accepted 23 July 2012

Academic Editor: Sadhan C. Jana

Copyright (C) 2012 Scott M. Berry et al. This is an open access article distributed under the Creative Commons Attribution License, which permits unrestricted use, distribution, and reproduction in any medium, provided the original work is properly cited.

Carbon-nanotube- (CNT-) doped polymer solutions were drawn into arrays of microfibers using a novel direct-write process. This process utilizes a micromanipulator-controlled syringe loaded with solvated polymer mixed with CNTs to "write" networks of composite fibers with precisely positioned endpoints. The diameters of these composite fibers are correlated to the degree of capillary thinning that occurs prior to the solidification of the directly written CNT-doped solution filament. The fibers had diameters ranging from $7 \mu \mathrm{m}$ to over $100 \mu \mathrm{m}$ and possessed conductivities as high as $0.1 \mathrm{Sm}^{-1}$. Fiber diameter was found to increase with increasing polymer concentration and decreasing fiber length and can be controlled through modulation of these parameters. The presence of CNTs was found not to significantly affect fiber diameter, despite the CNTs significant effect on viscosity, which was previously reported to influence diameter. This discrepancy is likely related to the non-Newtonian effects of CNT/polymer solutions, including an apparent shear thinning at increasing axial strain rates.

\section{Introduction}

CNT-doped polymers have emerged as popular materials in the production of microelectronic components and devices. These composites possess distinct attributes characteristic of both CNTs (high strength and electrical conductivity) and polymers (light weight, flexible, and customizable), making them ideal selections for the production of a variety of devices including chemical or mechanical sensors [1], flexible electromagnetic interference (EMI) shielding [2], and "smart" textiles [3]. One critical quality essential to the functionality of all microelectronic applications is the existence of sufficient conductivity throughout the composite material. Several groups have reported that conductivity of a $\mathrm{CNT} /$ polymer composite will increase with increasing CNT concentration in a nonlinear or nonisotropic manner [4-7]. The complex relationship between CNT concentration and conductivity is related to the principle of "percolation," which predicts a sharp rise in conductivity when CNT concentration surpasses a threshold value where a continuous network of CNTs exists within the surrounding polymer matrix. Furthermore, processing of composites into fiber geometries has been reported to align the CNTs along the fiber axis $[5,6]$. However, the effect of this alignment on conductivity seems unclear, as indicated by conflicting reports $[6,7]$ in the literature.

Micro/nanofibrous architectures have proven effective in a variety of microelectronic applications due to intrinsic advantages associated with fibrous structures including improved flexibility, maximum surface area, and increased strength. Melt spinning has emerged as a popular technique to process these bulk composites into fibers, with reported generation of fibers composed of CNT-doped polyamide 12 [8], polyamide 6 [9], PMMA [10], and polypropylene [11]. Additionally, CNT/polyvinyl alcohol (PVAc) composite fibers have been produced via wet spinning $[3,12]$ and CNT-doped poly(lactide) (PLA) [13] and polystyrene [14] via electrospinning. In recent work by Pabba et al. $[5,15]$, 


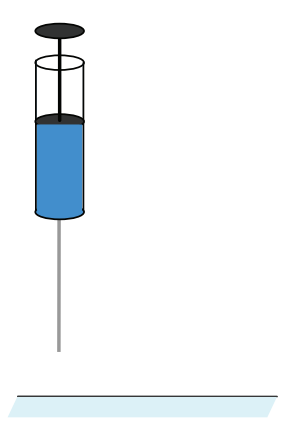

(a)

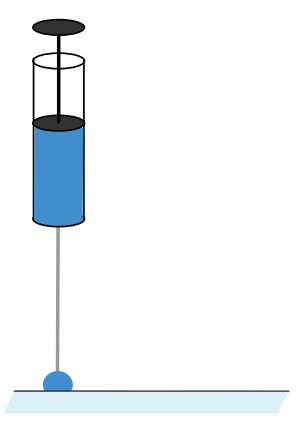

(b)

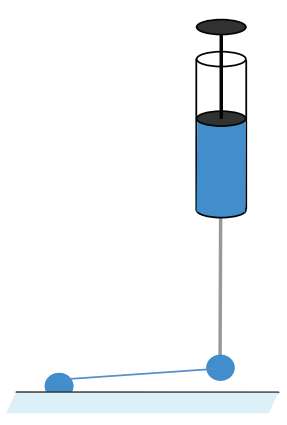

(c)

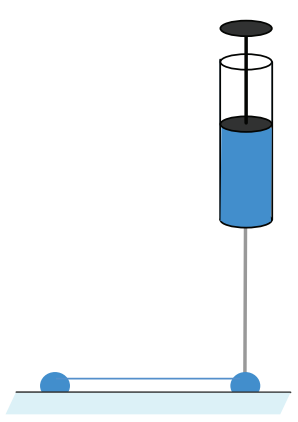

(d)

FIGURE 1: Direct-write drawing of CNT-doped polymer fibers. (a) Load syringe with CNT-doped solvated polymer. (b) Pressurize syringe to expel solution into contact with substrate. (c) Translate syringe to desired endpoint. (d) Pressurize syringe to establish second contact point.

a technique was reported in which viscous CNT/PMMA solutions were manually brushed across microfabricated pillar arrays to form networks of micro- and nanoscale solution filaments, which solidified to form suspended fibers. While each of these fiber fabrication strategies enables production of large quantities of fibers, none facilitates the construction of individual fibers that can be precisely positioned on any three-dimensional substrate. This limitation imposes constraints on the design of next-generation microelectronics, particularly those with 3D features.

In this investigation, CNT-doped polymer fibers that were precisely positioned in $3 \mathrm{D}$ were produced using a previously described direct-write technique $[16,17]$. The directwrite system consists of a micromanipulator-controlled syringe that was programmed to pattern arrays of fibers on a substrate. The direct-write syringe was first loaded with a test solution and positioned $500 \mu \mathrm{m}$ above a glass substrate (Figure 1(a)). The syringe was then pressurized to expel a pendant droplet of solution, which contacts and adheres to the substrate (Figure 1(b)). Next, the syringe was lifted $1 \mathrm{~mm}$ and translated laterally to extend this droplet into a filament of solution (Figure 1(c)). The fiber endpoint was established by lowering the syringe to its initial height after the translation, thereby allowing the pendant drop to make an additional contact point (Figure 1(d)). Meanwhile, during extension, the solution filament underwent surface tension-driven capillary thinning, while solvent evaporation promoted the solidification of the composite fiber. By repeating this process, arrays of composite fibers were created in series in a "connect-the-dots" manner.

In our previous work, we demonstrated that the diameters of the fibers with the direct-write system from polymer solutions alone (i.e., no CNTs) were dependent on both solution properties and direct-write system parameters. Specifically, fiber diameter increased with increasing viscosity, increasing solvent evaporation rate, decreasing surface tension, decreasing fiber length, and decreasing fiberdrawing rate $[16,17]$. Unfortunately, the applicability of these relationships is limited by the assumption that the polymer solution behaves in a "Newtonian-like" manner (i.e., that the capillary velocity of the solution, defined as the ratio of surface tension to viscosity, remains constant over the course of direct-write processing). Although some polymer solutions were found to meet this criterion, this critical assumption represents a significant shortcoming for the previously described model. Therefore, the purpose of the current investigation is to extend the direct-write technique to CNT/polymer composites and determine how the non-Newtonian nature of these materials affects directwrite outcomes.

\section{Materials and Methods}

2.1. Solution Preparation. Solutions containing $1 \%$ CNTs were prepared by dissolving either $24 \%$ or $26 \%$ (by wt.) poly(methyl methacrylate) (PMMA) (Sigma-Aldrich, St. Louis, MO, MW $=996,000 \mathrm{~g} / \mathrm{mol}$ ) in a solution of chlorobenzene supplemented with multiwalled nanotubes (MWNTs) (Conc. $=10 \mathrm{mg} / \mathrm{mL}$ ) that were functionalized to remain welldispersed in chlorobenzene (Zyvex NanoSolve, Richardson, TX). Once dried, the $24 \%$ and $26 \%$ solutions would produce solid PMMA with $4.0 \%$ or $3.7 \%$ CNTs, respectively. Control solutions containing no CNTs were prepared by dissolving 24 or 26\% (by wt.) PMMA in pure chlorobenzene.

2.2. Solution Characterization. The effect of axial strain on extensional viscosity was determined for PMMA solutions with and without the addition of CNTs. A capillary breakup rheometer (CaBER) (Thermo Haake 1, Thermo Electron Corp.) was utilized to measure the midpoint diameters, $D_{\text {mid }}(t)$, of filaments of each of the test solutions as they experienced surface tension-driven thinning. These filaments were formed by stretching cylinders of each solution in a near-instantaneous manner $\left(t_{\text {stretch }}=20 \mathrm{~ms}\right)$ from a length of $2 \mathrm{~mm}$ to a length of $8 \mathrm{~mm}$. The midpoint diameters of the elongated filaments were continuously monitored for 10 seconds with a laser micrometer at a sampling rate of $3,000 \mathrm{~Hz}$.

The midpoint diameter data collected by the CaBER was analyzed in order to determine the axial strain rate, $\dot{\varepsilon}$, of 
the solution filaments. Assuming uniaxial extension, the axial strain rate is correlated to midpoint diameter by [18]:

$$
\dot{\varepsilon}=\frac{-2}{D_{\text {mid }}(t)} \frac{d D_{\text {mid }}(t)}{d t} .
$$

This strain rate was then employed to calculate the apparent extensional viscosity, $\eta_{\text {ext }}$, of the test solutions using [19]:

$$
\eta_{\text {ext }}=\frac{(4 \cdot 0.7127-2) \sigma}{D_{\text {mid }}(t) \dot{\varepsilon}} .
$$

$\eta_{\text {ext }}$ was plotted as a function of axial strain rate over the course of the CaBER experiments to determine the level of dependency of extensional viscosity on strain rate. The dependency of $\eta_{\text {ext }}$ on $\dot{\varepsilon}$ was quantified to determine if any extensional thinning (or thickening) of the test solutions was apparent, suggesting non-Newtonian behavior during extensional flow [19]. Surface tension was measured via the Wilhelmy technique [20], and solvent mass transfer coefficient was measured using thermogravimetric analysis (TGA), as previously described [16].

2.3. Direct-Write Fiber Fabrication. Fibers were drawn to three different lengths $(4,8$, and $16 \mathrm{~mm})$ from each of the test solutions at a rate of $20 \mathrm{~mm} / \mathrm{s}$, which was found to work well in previous studies [16, 21]. A total of 8 drawing attempts were made for each combination of solution and length. The syringe was pressurized to expel a constant flow of approximately $20 \mu \mathrm{L} / \mathrm{min}$ through a 22 gauge needle (I.D. $=394 \mu \mathrm{m})$. After drawing, each array was coated with a thin layer $(t=10$ to $20 \mathrm{~nm})$ of gold/palladium alloy and the diameter of each fiber was measured in three locations (at the midpoint and $200 \mu \mathrm{m}$ from each end) using a scanning electron microscope (SEM) (Zeiss Supra 35VP, Thornwood, NY). Process yield was determined by calculating the ratio of unbroken fibers to fiber drawing attempts.

2.4. Electrical Transport Measurement. The conductivity of the MWNT-doped fibers was measured to quantify the electrical transport along the fibers. The ends of each fiber were metalized with silver paint to minimize contact resistance, and the resistance of each fiber was analyzed with a highly sensitive parameter analyzer (Agilent 4156, Santa Clara, CA). Conductivity for each fiber was calculated by

$$
G=\frac{4 L}{R \pi\left(D_{\mathrm{avg}}\right)^{2}},
$$

where $G$ is conductivity, $L$ is the length of the fiber, $R$ is the measured fiber resistance, and $D_{\text {avg }}$ is the average fiber diameter [22].

2.5. Cross-Sectional Imaging. Cross-sections of fibers from each test solution were inspected with an SEM to determine the homogeneity of the fibers. Fibers were frozen in liquid nitrogen for 1 minute, cleaved with a razor blade, and coated with a thin layer ( $t=10$ to $20 \mathrm{~nm}$ ) of gold-palladium prior to imaging. The images were then used to determine the level and distribution of CNT aggregation within the fibers.

\section{Results and Discussion}

3.1. Solution Characterization. Solutions containing PMMA and CNTs were observed to possess surface tensions that were slightly higher than pure chlorobenzene $(\sigma=33.28 \mathrm{mN} / \mathrm{m}$ at $20^{\circ} \mathrm{C}$ ). The presence of CNTs in the solutions was found to have no significant effect on the surface tensions of the PMMA solutions (Table 1). The inclusion of CNTs in the test solutions was found to impede the evaporation of the chlorobenzene from the solutions as evidenced by the lower values in the mass transfer coefficients.

The CaBER was utilized to instantaneously extend volumes of each test solution into liquid bridges with initial aspect ratios $(L / D)$ of approximately 2.7. The diameters of these liquid bridges, $D_{\text {mid }}(t)$, were monitored for 10 seconds as surface tension-driven thinning occurred (Figure 2(a)). This figure illustrates that solutions with no CNTs were observed to thin more rapidly than those with $1 \%$ CNTs and the 24\% PMMA solution with no CNTs thinned faster than the corresponding 26\% PMMA solution. Both solutions with $1 \%$ CNTs thinned at approximately the same rate, suggesting that the presence of CNTs is the dominating factor in the surface tension-driven thinning of these solutions. The measurement of $D_{\text {mid }}(t)$ was used with (1) and (2) and the data from Table 1 to calculate $\dot{\varepsilon}$ and $\eta_{\text {ext }}$, which are plotted against each other in Figure 2(b). Unfortunately, the CaBER instrument was only able to apply a limited range of axial strain rates to the test solutions and this range was different for each solution (i.e., each of the curves in Figure 2(b) is for a small range of strain rate, making comparison between solutions more difficult). However, based on this limited data, it appears that the CNT composites experience shear thinning while the extensional viscosity of the PMMA-only solutions is relatively independent of shear rate.

3.2. Direct-Write Production of Fibers. The direct-write process was successfully employed to produce CNT-doped fibers with diameters ranging from $7 \mu \mathrm{m}$ to over $100 \mu \mathrm{m}$ (Figure 3). The fiber diameter data is given in Figure 4(a). ANOVA analysis of this data determined that fiber diameter increased with increasing PMMA concentration $(P<0.001)$ and decreasing length $(P<0.001)$. However, the presence of $1 \%$ CNTs did not significantly influence fiber diameter $(P=0.2)$ except for the $4 \mathrm{~mm}$ long fibers drawn from the $24 \%$ polymer solution $(P<0.005)$. Paired $t$-Test analysis confirmed that the fibers possessed significantly smaller diameters midway along their lengths relative to their ends $(P<0.001)$. Also, the ends of the fibers where drawing was begun were significantly smaller than the ends where drawing was completed $(P<0.001)$. The average coefficient of variation (diameter standard deviation/diameter average) for all fibers along the length of the fibers was $34 \%$. The process yield for the direct-write process exceeded $85 \%$ under all test conditions (Figure 4(b)).

The characterization of test solution rheology suggests that the CNT-doped solutions experience strain thinning when exposed to axial strain, whereas nondoped solutions behave in a more Newtonian manner, even at higher strain rates (Figure 2(b)). A similar trend was observed 


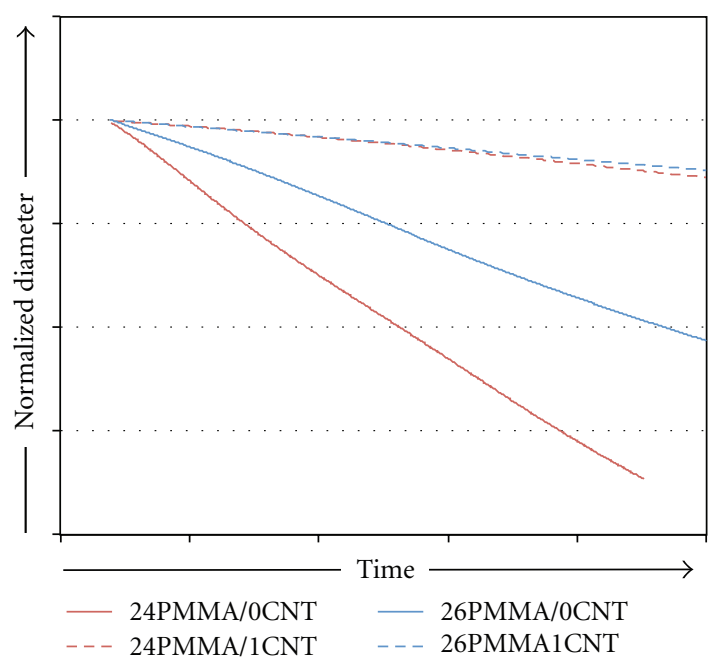

(a)

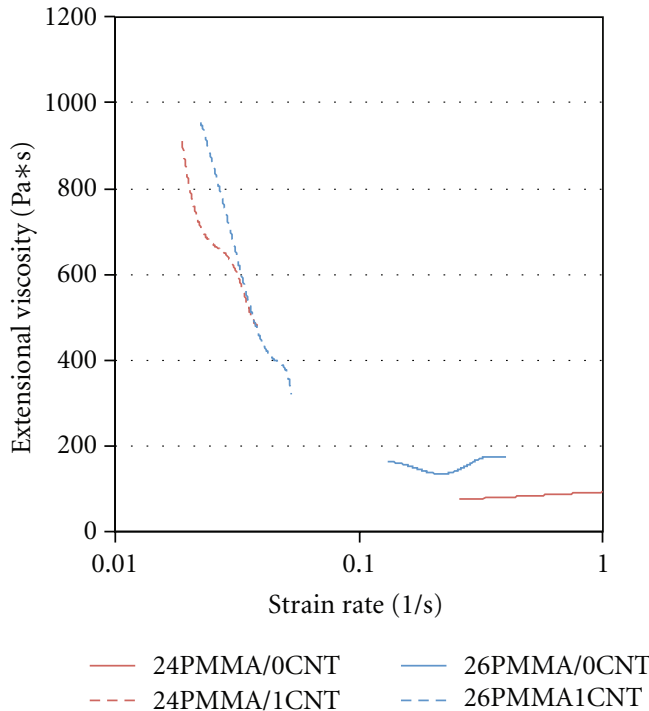

(b)

FIGURE 2: (a) CaBER data illustrating the surface tension-driven thinning of the test solutions as a function of time. (b) Extensional viscosity of test solutions as a function of axial strain rate experienced during capillary thinning.

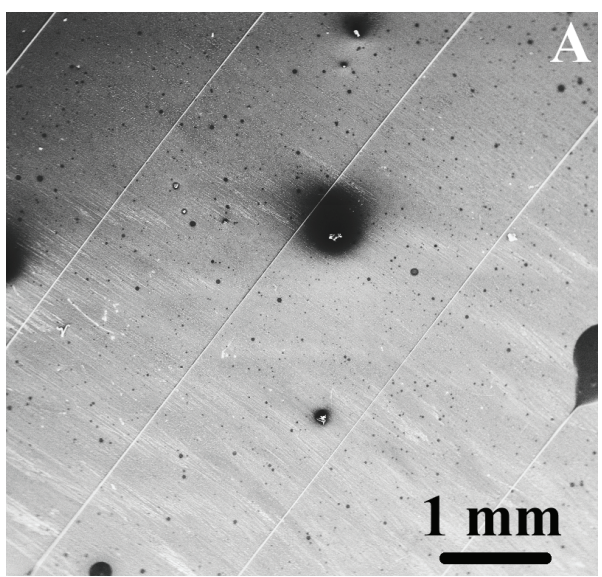

(a)

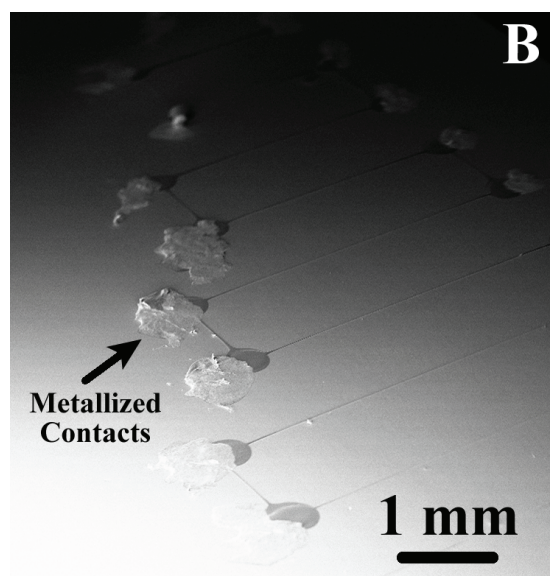

(b)

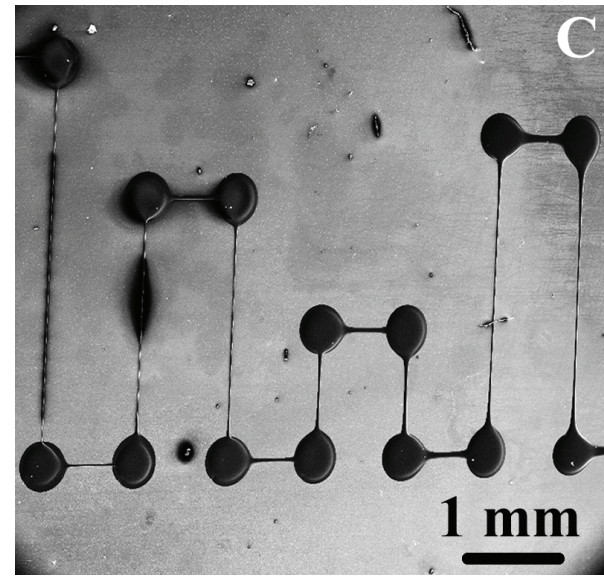

(c)

FIGURE 3: Fibers produced with the direct-write process. (a) Multiple parallel fibers. (b) Ends of fibers metalized with silver paste. (c) Test array of fibers with varying lengths. Fibers appear darker in (c) due to differences in SEM imaging contrast. 
TABLE 1: Surface tensions and mass transfer coefficients of test solutions.

\begin{tabular}{lccc}
\hline $\begin{array}{l}\text { PMMA solution } \\
\text { concentration }\end{array}$ & $\begin{array}{c}\text { CNT solution } \\
\text { concentration }\end{array}$ & $\begin{array}{c}\text { Surface tension } \\
(\mathrm{mN} / \mathrm{m})\end{array}$ & $\begin{array}{c}\text { Mass transfer } \\
\text { coefficient }(\mathrm{m} / \mathrm{s})\end{array}$ \\
\hline $24 \%$ & $0 \%$ & $37.2 \pm 7.3$ & $1.06 E-07$ \\
$26 \%$ & $0 \%$ & $43.6 \pm 7.2$ & $1.06 E-07$ \\
$24 \%$ & $1 \%$ & $45.8 \pm 5.2$ & $5.82 E-08$ \\
$26 \%$ & $1 \%$ & $45.3 \pm 1.5$ & $5.82 E-08$ \\
\hline
\end{tabular}

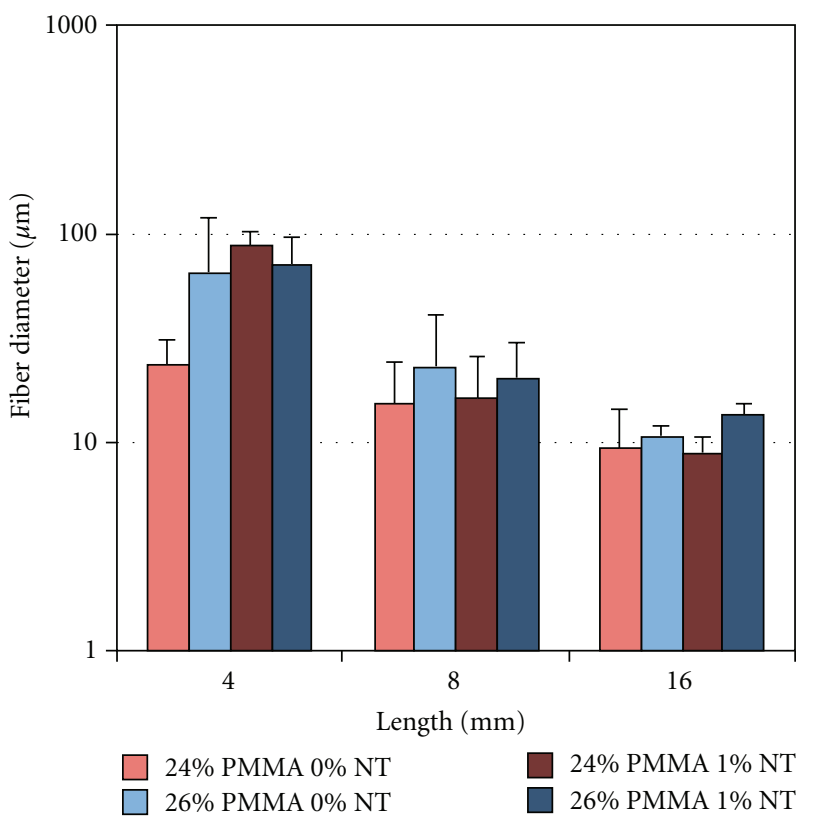

(a)

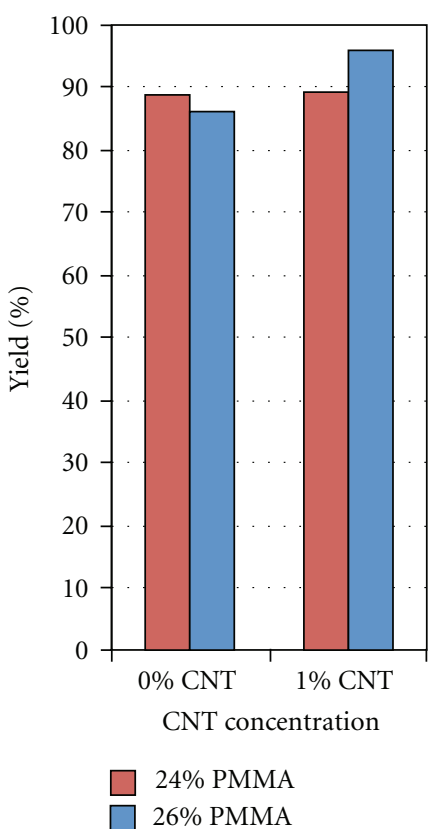

(b)

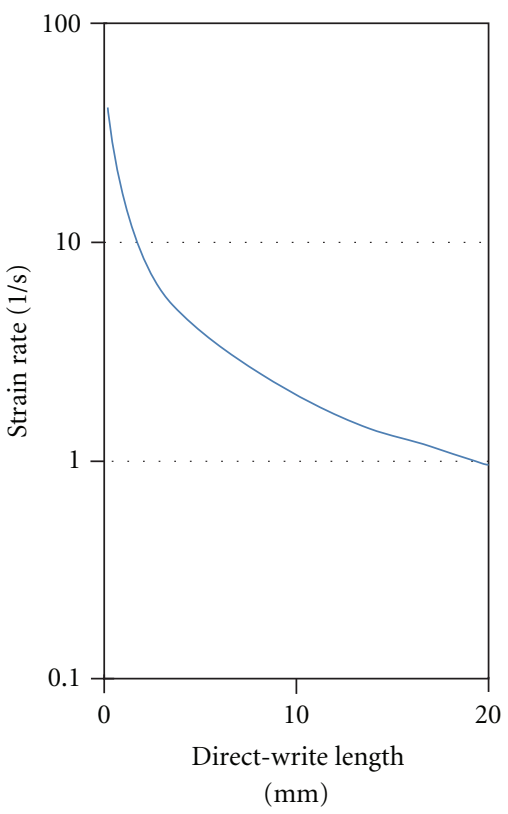

(c)

FIGURE 4: Fiber diameter as a function of fiber length, PMMA concentration, CNT concentration. Error bars denote standard deviation and $n=8$. (b) Direct-write yield as a function of PMMA concentration and CNT concentration. (c) Axial strain imparted on the solution by the motion of the direct-write system as a function of drawing length, as predicted by (4). 


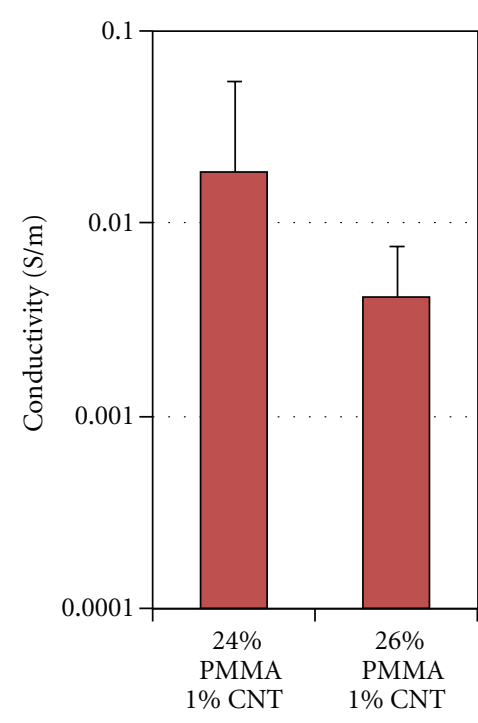

(a)

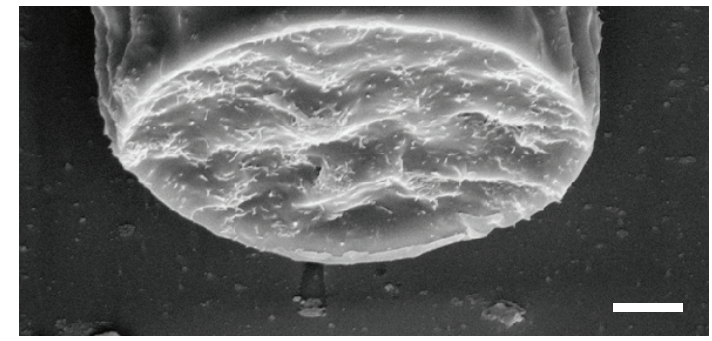

(b)

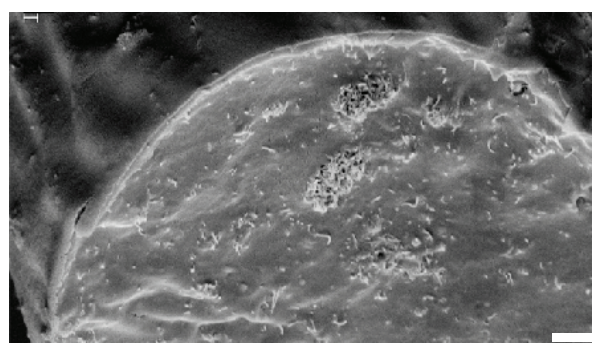

(c)

Figure 5: (a) Conductivity as a function of PMMA concentration. (b) Cross-sectional image of fiber drawn from 24\% PMMA/1\% CNT solution. (c) Cross-sectional image of fiber drawn from 26\% PMMA/1\% CNT solution. Scale bars in (b) and (c) are $1 \mu \mathrm{m}$.

by Rahatekar et al. who concluded that epoxy doped with CNTs will thin when exposed to increasing shearing strain rates until it asymptotically approaches the viscosity of pure epoxy [23]. Although the CaBER system was not capable of inducing high axial strain rates $(\dot{\varepsilon}>0.1)$ on the CNTdoped solutions, the orientations of the curves in Figure 2(b) suggests that such an asymptotic relationship may exist (i.e., the extensional viscosity of the CNT-doped solutions could approach that of the pure PMMA solutions if axial strain rate was increased).

During direct-write drawing, the solution experiences axial strain due to surface tension-driven thinning in a manner similar to the CaBER experiments. However, the motion of the direct-write syringe, which imposed additional axial strain on the solution, augmented this strain. The magnitude of this strain generated by the motion of the direct-write syringe, $\varepsilon_{\mathrm{DW}}$, is given by [24]:

$$
\varepsilon_{\mathrm{DW}}=\ln \left(\frac{L(t)}{L_{0}}\right)
$$

where $L(t)$ is the length of the direct-write fiber as it is drawn and $L_{0}$ is the initial length of the solution droplet on the direct-write syringe before drawing $\left(L_{0} \approx 394 \mu \mathrm{m}\right)$.
Equation (4) is differentiated to obtain the axial strain rate imparted by the direct-write process:

$$
\dot{\varepsilon}_{\mathrm{DW}}=\frac{1}{L(t)} \frac{d L(t)}{d t}
$$

where $d L(t) / d t$ is the drawing velocity of the direct-write system. Figure 4(c) illustrates the magnitude of the axial strain rate generated by the direct-write process for fibers drawn up to lengths of $16 \mathrm{~mm}$ at a test velocity of $20 \mathrm{~mm} / \mathrm{s}$. Comparison of these axial strain rates with those generated by the CaBER (Figure 2(b)), which expresses the magnitude of axial strain rate during surface tension-driven thinning only, suggest that the motion of the drawing process influences axial strain rate by an approximate order of magnitude more than the surface tension-driven thinning motion alone. Furthermore, the axial strain rates encountered during the direct-write process may be sufficiently large to thin the CNT-doped solutions into the asymptotic regime where $\eta_{\text {ext }}$ approaches $\eta_{\text {ext }}$ of the homopolymer system, providing a potential explanation as to why CNT concentration was shown not to influence fiber diameter, except in the case of the shortest fibers (i.e., lowest applied strain) with the lower polymer concentration (i.e., more thinning of the undoped solution). 
3.3. Fiber Conductivity. It was observed that conductivity decreases with increasing PMMA concentration (Figure 5(a)), but it was not statistically significant $(P=0.1)$. Fiber length was found not to significantly affect conductivity, suggesting that lengthening fibers (from $4 \mathrm{~mm}$ to $16 \mathrm{~mm}$ ) did not induce a major change in the $\mathrm{CNT} /$ polymer matrix architecture. Cross-sectional imaging of representative fibers drawn from each test solution illustrated the level of CNT dispersion within the fibers, as shown in Figures 5(b) and 5(c). In general, the magnitude of conductivity was similar to that previously reported for PMMA/CNT composite fibers [4].

\section{Conclusions}

Arrays of CNT-doped PMMA fibers were successfully produced using the direct-write method. This development enables production of conductive composite fibers that are precisely positioned, thus facilitating the fabrication of point-to-point connecters in situ. Directly written composite fibers possessed diameters ranging from $7 \mu \mathrm{m}$ to over $100 \mu \mathrm{m}$ and were typically produced with process yields greater than $85 \%$. Fiber diameter increased with increasing PMMA concentration and decreasing fiber length. Analysis of the rheological data indicated that the CNT-doped solutions experienced significant reduction in extensional viscosity when exposed to strain rates consistent with those imposed by the direct-write process. Conductivity was not significantly affected by PMMA concentration or fiber length and fibers with conductivities as high as $0.1 \mathrm{Sm}^{-1}$ were fabricated.

\section{Acknowledgments}

The work presented in this paper was funded by the National Science Foundation NIRT Program (ECS-0506941), NSF PFI Program (EEC-0438604), and National Aeronautics and Space Administration cooperative agreement (NCC5-571).

\section{References}

[1] S. M. Cho, Y. J. Kim, Y. S. Kim, Y. Yang, and S. C. Ha, “The application of carbon nanotube-polymer composite as gas sensing materials," in Proceedings of the IEEE Sensors, pp. 701704, October 2004

[2] Y. Yang, M. C. Gupta, and K. L. Dudley, "Towards costefficient EMI shielding materials using carbon nanostructurebased nanocomposites," Nanotechnology, vol. 18, no. 34, Article ID 345701, 2007.

[3] A. B. Dalton, S. Collins, E. Muñoz et al., "Super-tough carbonnanotube fibres," Nature, vol. 423, no. 6941, p. 703, 2003.

[4] J. M. Benoit, B. Corraze, S. Lefrant, W. J. Blau, P. Bernier, and O. Chauvet, "Transport properties of PMMA-carbon nanotubes composites," Synthetic Metals, vol. 121, no. 1-3, pp. 1215-1216, 2001.

[5] S. Pabba, A. N. Sidorov, S. M. Berry et al., "Oriented nanomaterial air bridges formed from suspended polymercomposite nanofibers," ACS Nano, vol. 1, no. 1, pp. 52-67, 2007.

[6] F. Du, J. E. Fischer, and K. I. Winey, "Coagulation method for preparing single-walled carbon nanotube/poly(methyl methacrylate) composites and their modulus, electrical conductivity, and thermal stability," Journal of Polymer Science, Part B, vol. 41, no. 24, pp. 3333-3338, 2003.

[7] R. Haggenmueller, H. H. Gommans, A. G. Rinzler, J. E. Fischer, and K. I. Winey, "Aligned single-wall carbon nanotubes in composites by melt processing methods," Chemical Physics Letters, vol. 330, no. 3-4, pp. 219-225, 2000.

[8] J. K. W. Sandler, S. Pegel, M. Cadek et al., "A comparative study of melt spun polyamide- 12 fibres reinforced with carbon nanotubes and nanofibres," Polymer, vol. 45, no. 6, pp. 20012015, 2004.

[9] J. Gao, M. E. Itkis, A. Yu, E. Bekyarova, B. Zhao, and R. C. Haddon, "Continuous spinning of a single-walled carbon nanotube-nylon composite fiber," Journal of the American Chemical Society, vol. 127, no. 11, pp. 3847-3854, 2005.

[10] J. Zeng, B. Saltysiak, W. S. Johnson, D. A. Schiraldi, and S. Kumar, "Processing and properties of poly(methyl methacrylate)/carbon nano fiber composites," Composites Part B, vol. 35, no. 2, pp. 173-178, 2004.

[11] S. Kumar, H. Doshi, M. Srinivasarao, J. O. Park, and D. A. Schiraldi, "Fibers from polypropylene/nano carbon fiber composites," Polymer, vol. 43, no. 5, pp. 1701-1703, 2002.

[12] A. B. Dalton, S. Collins, J. Razal et al., "Continuous carbon nanotube composite fibers: properties, potential applications, and problems," Journal of Materials Chemistry, vol. 14, no. 1, pp. 1-3, 2004.

[13] F. Ko, Y. Gogotsi, A. Ali et al., "Electrospinning of continuous carbon nanotube-filled nanofiber yarns," Advanced Materials, vol. 15, no. 14, pp. 1161-1165, 2003.

[14] R. Sen, B. Zhao, D. Perea et al., "Preparation of single-walled carbon nanotube reinforced polystyrene and polyurethane nanofibers and membranes by electrospinning," Nano Letters, vol. 4, no. 3, pp. 459-464, 2004.

[15] S. Pabba, S. M. Berry, M. M. Yazdapanah, R. S. Keynton, and R. W. Cohn, "Nanotube suspension bridges directly fabricated from nanotube-polymer suspensions by manual brushing," in Proceedings of the 6th IEEE Conference on Nanotechnology (IEEE-NANO '06), pp. 565-568, Cincinnati, Ohio, USA, June 2006.

[16] S. M. Berry, S. D. Cambron, S. P. Warren, S. Pabba, R. W. Cohn, and R. S. Keynton, "Characterization and modeling of direct-write fabrication of microscale polymer fibers," Polymer, vol. 52, no. 7, pp. 1654-1661, 2011.

[17] S. M. Berry, S. A. Harfenist, R. W. Cohn, and R. S. Keynton, "Characterization of micromanipulator-controlled dry spinning of micro- and sub-microscale polymer fibers," Journal of Micromechanics and Microengineering, vol. 16, no. 9, pp. 18251832, 2006.

[18] A. Tripathi, P. Whittingstall, and G. H. McKinley, "Using filament stretching rheometry to predict strand formation and "processability" in adhesives and other non-Newtonian fluids," Rheologica Acta, vol. 39, no. 4, pp. 321-337, 2000.

[19] Thermo Electron Corp, CaBER Instruction Manual.

[20] P. G. de Gennes, F. Brochard-Wyart, and D. Quere, Capillarity and Wettuing Phenomena: Drops, Bubbles, Pearls, Waves, Springer, Berlin, Germany, 2004.

[21] S. M. Berry, S. P. Warren, D. A. Hilgart et al., "Endothelial cell scaffolds generated by 3D direct writing of biodegradable polymer microfibers," Biomaterials, vol. 32 , no. 7, pp. 18721879, 2011.

[22] D. Halliday, R. Resnick, and J. Walker, Fundamentals of Physics, vol. 1, John Wiley \& Sons, Hoboken, NJ, USA, 7th edition, 2005. 
[23] S. S. Rahatekar, K. K. K. Koziol, S. A. Butler et al., "Optical microstructure and viscosity enhancement for an epoxy resin matrix containing multiwall carbon nanotubes," Journal of Rheology, vol. 50, no. 5, pp. 599-610, 2006.

[24] M. Yao and G. H. McKinley, "Numerical simulation of extensional deformations of viscoelastic liquid bridges in filament stretching devices," Journal of Non-Newtonian Fluid Mechanics, vol. 74, no. 1-3, pp. 47-88, 1998. 



The Scientific World Journal

Submit your manuscripts at

http://www.hindawi.com

\section{World Journal}

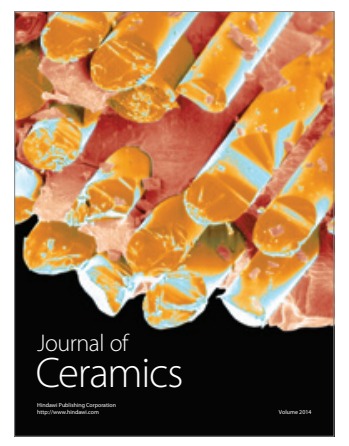

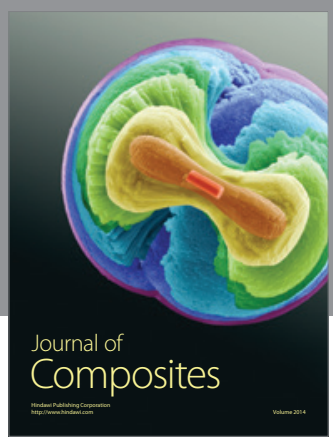
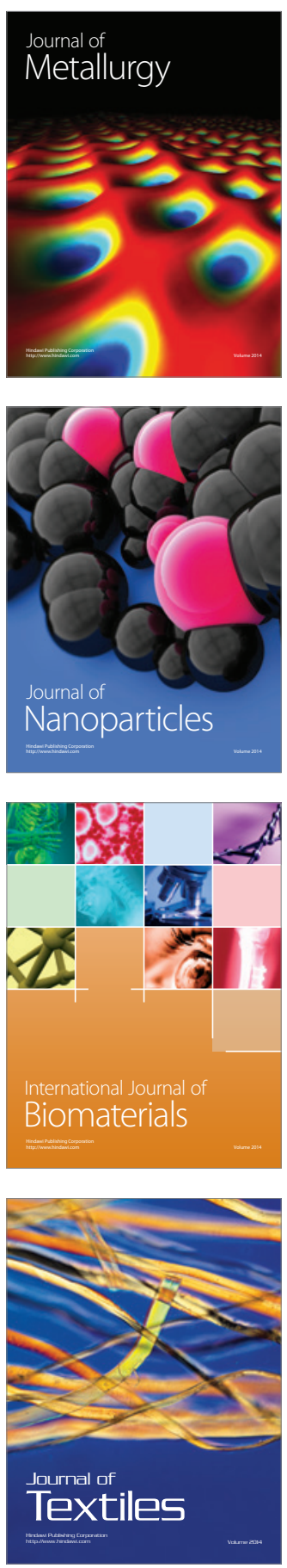